TITLE:

\title{
Frequency domain optical tomography using a Monte Carlo perturbation method
}

$\operatorname{AUTHOR}(S):$

Yamamoto, Toshihiro; Sakamoto, Hiroki

CITATION:

Yamamoto, Toshihiro ...[et al]. Frequency domain optical tomography using a Monte Carlo perturbation method. Optics Communications 2016, 364: 165-176

\section{ISSUE DATE:}

2016-04

URL:

http://hdl.handle.net/2433/203606

\section{RIGHT:}

(C) 2015. This manuscript version is made available under the CC-BY-NC-ND 4.0 license

http://creativecommons.org/licenses/by-nc-nd/4.0/; The full-text file will be made open to the public on 1 April 2018 in accordance with publisher's 'Terms and Conditions for Self-Archiving'.; This is not the published version. Please cite only the published version; この論文は出版社版でありません。引用の際には出版社版をご確認ご利用ください。 


\section{Frequency Domain Optical Tomography Using a Monte Carlo Perturbation Method}

\section{Abstract}

A frequency domain Monte Carlo method is applied to near-infrared optical tomography, where an intensity-modulated light source with a given modulation frequency is used to reconstruct optical properties. The frequency domain reconstruction technique allows for better separation between the scattering and absorption properties of inclusions, even for ill-posed inverse problems, due to cross-talk between the scattering and absorption reconstructions. The frequency domain Monte Carlo calculation for light transport in an absorbing and scattering medium has thus far been analyzed mostly for the reconstruction of optical properties in simple layered tissues. This study applies a Monte Carlo calculation algorithm, which can handle complex-valued particle weights for solving a frequency domain transport equation, to optical tomography in two-dimensional heterogeneous tissues. The Jacobian matrix that is needed to reconstruct the optical properties is obtained by a first-order "differential operator" technique, which involves less variance than the conventional "correlated sampling" technique. The numerical examples in this paper indicate that the newly proposed Monte Carlo method provides reconstructed results for the scattering and absorption coefficients that compare favorably with the results obtained from conventional deterministic or Monte Carlo methods.

\footnotetext{
* Corresponding author. Tel: +81 72451 2414; Fax: +81 724512658 E-mail: toshihiro.yamamoto223@gmail.com (T.Yamamoto)
} 
Keywords: Monte Carlo; frequency domain; optical tomography; perturbation; ART

\section{Introduction}

Near-infrared optical tomography has been developed as a promising technique for the 5 effective retrieval of spatially dependent optical parameters in a turbid medium, such as 6 absorption and scattering coefficients. The technique reconstructs the optical parameters of 7 an internal medium from the measured transmittance and reflectance at the boundary 8 surface of a probed medium. The propagation of radiation in a medium is mainly 9 calculated using the diffusion approximation theory or the exact radiation transport theory. 10 It is well known that the diffusion approximation theory introduces inaccuracy in void-like 11 regions, near the source, or at the boundary surface, but it is very cost-effective and easy to 12 solve $[1,2]$. Representative methods for solving a radiative transfer equation include the 13 discrete ordinates method and the Monte Carlo method. The research performed thus far 14 has utilized the discrete ordinates method for the purpose of near-infrared optical 15 tomography [1-13]. Some spatial discretization schemes, such as the finite volume or finite 16 difference scheme, are commonly used and are associated with the discrete ordinates 17 method. The finite element method has drawn increasing attention due to its flexibility in 18 handling complex geometries.

19 Another promising calculation tool proposed here is the Monte Carlo method. The most notable advantage of the Monte Carlo method over deterministic methods is its lack of limitations when handling three-dimensional complex geometries. The Monte Carlo method also eliminates discretization in the dimensions of energy, time and angle. If a large number of particles are used, the Monte Carlo method is therefore free from the ray effects and false scattering that are major sources of inaccuracy in the discrete ordinates method. The disadvantage of the Monte Carlo method is that an estimate is always accompanied by statistical uncertainty and the wave properties of light is ignored. In general, the Monte Carlo method is thought to be overly time-consuming and expensive for reducing uncertainties below an acceptable level. However, with the advent of high 
1 performance CPUs, massive parallel computing technologies, and some of new Monte

2 Carlo methods that are being developed, the drawbacks of the Monte Carlo method can be

3 overcome, even for problems that were previously impossibly time-consuming.

Radiation transfer calculations for optical tomography are often performed in the frequency domain. In a frequency domain calculation, the radiation beam intensity is 6 modulated in amplitude at a given frequency. By using a modulated radiance, phase 7 information is available in addition to amplitude. When we seek to simultaneously reconstruct two optical coefficients, such as scattering and absorption coefficients, cross-talk between the two coefficients has been observed, and may lead to a wrong diagnosis [4]. To avoid cross-talk, additional information is needed. Frequency domain measurements would provide information regarding the phase of the radiation in addition to its intensity. Thus, frequency domain techniques allow for better separation of absorption and scattering effects [14].

The deterministic radiation transfer calculation method in the frequency domain is an established and common tool. To the best of our knowledge, there have not been many studies on the Monte Carlo method for optical tomography, especially in the frequency domain. A general survey is provided on the Monte Carlo modeling of radiation transfer in tissue optics in [15], which reviews the history and recent progress in the development of the Monte Carlo method in this area of research in detail. Some previous studies that performed frequency domain Monte Carlo calculations have been published in the literature [16-19]. In [16], the Monte Carlo method in the frequency domain is used to analyze the amplitude and phase delay of detected waves at a single frequency. This method is referred to as the "shortcut method" because a time domain calculation that includes many frequencies can be bypassed. The amplitude and phase at a single frequency can be measured or calculated using a temporal Fourier transform of the time series data for the detected signals. The shortcut method performs a Monte Carlo calculation only for a single modulation frequency. In $[17,18]$ radiative signals in the frequency domain are 
1 obtained via the Monte Carlo method following the techniques presented in [16], and 2 provide solutions to inverse photon migration problems in heterogeneous turbid media. In 3 [19], a spatial Fourier transform is performed on the radiative transfer equation to analyze 4 a layered tissue system. The complex radiation transport equation is solved via the Monte $5 \quad$ Carlo method using complex-valued particle weights.

There is another advantage when treating a radiative transfer equation in the frequency domain: the time-dependent radiative transfer equation can easily be solved in the frequency domain because no time-derivative term exists in the frequency domain equation $[20,21]$. At first, the time-dependent radiative transfer equation is transformed into the frequency domain equation by applying a Fourier transform. The complex-valued frequency domain equation is solved for each frequency that is contained in the incident light source. Time-dependent results in the time domain can be obtained by applying an inverse Fourier transform to the results from the frequency domain.

The authors recently applied this deterministic technique to solve a time-dependent neutron transport equation in a subcritical nuclear reactor [22] using the Monte Carlo method. The neutron transient behavior is induced by the time variation of the neutron source intensity. The complex-valued neutron transport equation in the frequency domain is solved for each frequency contained in the neutron source time variation to obtain the complex-valued neutron flux. By applying an inverse Fourier transform to the complex-valued neutron flux, the time variation of the neutron flux is obtained.

Previous studies performed by Hayakawa et al. [17], Seo et al. [23], Zhao et al., [24, 25] use the Monte Carlo method in the frequency domain for solving inverse problems to determine optical properties. A similar study was performed by Sharma et al. [26], not for a modulated source but for steady-state diffuse reflectance in two-layered phantoms. These studies address homogeneous tissue $[24,25]$ or heterogeneous simple geometries [26] that are composed of a one-dimensional, two-layered tissue. Kumar and Vasu [27], and Yalavarthy et al. [28] performed studies to reconstruct the optical properties of a 
heterogeneous two-dimensional tissue model using the perturbation Monte Carlo technique. Their studies do not address the frequency domain problem and are limited to steady-state Monte Carlo modeling. The study in this paper aims to extend the Monte Carlo calculation algorithm developed for nuclear reactor kinetics calculations in the frequency domain to transient radiative transfer calculations, which can subsequently be available for optical tomography. This will allow reconstruction of the multi-dimensional distribution of optical properties, as performed in [4-12] using the deterministic methods.

\section{Algorithm for the frequency domain Monte Carlo calculation method}

In this section, we briefly review the method adopted in [22] that outlines how to solve the radiative transfer equation in the frequency domain using the Monte Carlo method. The time-dependent radiative transfer equation in a non-multiplying medium is written as [22]

$$
\begin{aligned}
& \frac{1}{c} \frac{\partial}{\partial t} I(\mathbf{r}, \mathbf{\Omega}, t) \\
& =-\mathbf{\Omega} \cdot \nabla I(\mathbf{r}, \mathbf{\Omega}, t)-\left(\mu_{a}(\mathbf{r})+\mu_{s}(\mathbf{r})\right) I(\mathbf{r}, \mathbf{\Omega}, t) \\
& +\frac{\mu_{s}(\mathbf{r})}{4 \pi} \int_{4 \pi} d \mathbf{\Omega}^{\prime} f\left(\mathbf{r}, \mathbf{\Omega}^{\prime} \rightarrow \mathbf{\Omega}\right) I\left(\mathbf{r}, \mathbf{\Omega}^{\prime}, t\right) \\
& +S(\mathbf{r}, \mathbf{\Omega}, t),
\end{aligned}
$$

where $I(\mathbf{r}, \mathbf{\Omega}, t)=$ the radiant power per unit solid angle per unit area at position $\mathbf{r}$ with direction $\boldsymbol{\Omega}$ and time $t, c=$ the light speed within the medium, $\mu_{a}=$ the absorption coefficient, $\mu_{s}=$ the scattering coefficient, $f\left(\mathbf{r}, \mathbf{\Omega}^{\prime} \rightarrow \mathbf{\Omega}\right)=$ the angular distribution of the scattered radiation, and $S=$ the radiation source term. This study chooses the Henyey-Greenstein function for the angular distribution of the scattered radiation as [9]

$$
f\left(\mathbf{r}, \boldsymbol{\Omega}^{\prime} \rightarrow \mathbf{\Omega}\right)=\frac{1-g^{2}}{\left(1+g^{2}-2 g \cos \theta\right)^{3 / 2}},
$$

where $\theta$ is the angle between $\boldsymbol{\Omega}^{\prime}$ and $\boldsymbol{\Omega}$, and $g$ is the anisotropy factor.

The external collimated beam that is often used in optical tomography penetrates the medium and is scattered within the medium in the course of its penetration. Due to strong discontinuities, radiative transport calculations are conventionally performed in two steps. In the first step, the collimated intensity that obeys the extinction law is obtained. In the 
1 second step, the scattered intensity induced by the scattering of the collimated intensity is 2 obtained. However, for the Monte Carlo method, the discontinuity does not cause any 3 difficulties within a small-sized medium treated in optical tomography. Thus, the 4 collimated and scattered intensities are calculated simultaneously in this study.

The time domain equation, Eq. (1), is converted to a frequency domain equation via a Fourier transformation. We obtain the transport equation for the radiation intensity in the frequency domain [4-12]:

$$
\begin{aligned}
& \mathbf{\Omega} \cdot \nabla \tilde{I}(\mathbf{r}, \mathbf{\Omega}, \omega)+\left(\mu_{a}(\mathbf{r})+\mu_{s}(\mathbf{r})\right) \tilde{I}(\mathbf{r}, \mathbf{\Omega}, \omega) \\
& =\frac{\mu_{s}(\mathbf{r})}{4 \pi} \int_{4 \pi} d \mathbf{\Omega}^{\prime} f\left(\mathbf{r}, \boldsymbol{\Omega}^{\prime} \rightarrow \boldsymbol{\Omega}\right) \tilde{I}\left(\mathbf{r}, \mathbf{\Omega}^{\prime}, \omega\right) \\
& -\frac{i \omega}{c} \tilde{I}(\mathbf{r}, \boldsymbol{\Omega}, \omega)+\tilde{S}(\mathbf{r}, \boldsymbol{\Omega}, \omega),
\end{aligned}
$$

$$
\begin{gathered}
\tilde{I}(\mathbf{r}, \boldsymbol{\Omega}, \omega) \equiv \int_{-\infty}^{+\infty} I(\mathbf{r}, \boldsymbol{\Omega}, t) e^{-i \omega t} d t \\
\tilde{S}(\mathbf{r}, \boldsymbol{\Omega}, \omega) \equiv \int_{-\infty}^{+\infty} S(\mathbf{r}, \boldsymbol{\Omega}, t) e^{-i \omega t} d t .
\end{gathered}
$$

The tilde denotes a complex-valued quantity. Eq. (3) is a fixed source equation in the frequency domain. The algorithm to obtain $\tilde{I}(\mathbf{r}, \mathbf{\Omega}, \omega)$ using Eq. (3), which explicitly uses complex-valued weights, was established in our previous publications by the authors [22, 29-32] and is briefly presented again here. Hereafter, the word "particle" is used to stand for "photon particle".

(1) First, a time-dependent source intensity is converted to a Fourier transformation using Eq. (5) to obtain $\tilde{S}(\mathbf{r}, \boldsymbol{\Omega}, \omega)$. Here, we consider an intensity-modulated source with a fixed frequency. Thus, $\omega$ is equal to the modulation frequency. A source particle is emitted from the source position. The position and angle of the source particle is determined from the probability density function of $\tilde{S}(\mathbf{r}, \mathbf{\Omega}, \omega)$. The weight of the source particle is given in proportion to the source intensity at $(\mathbf{r}, \mathbf{\Omega})$. If we choose a cosine wave as the modulated source intensity, the weight of the source particle is a real value. On the other hand, the weight has an imaginary value if a sinusoidal wave is chosen. 
1 (2) The particle is displaced to the next collision site along the flight direction. The flight 2 distance $s$ is determined by $s=-\ln \xi / \beta$, where $\xi$ is a uniform pseudo random number 3 from $(0,1]$ and $\beta=\mu_{a}+\mu_{s}$. The determination of the next collision site is the same as the 4 conventional Monte Carlo method. If the flight distance $s$ is longer than the distance to the 5 boundary of the region where the optical properties are constant, the flight distance beyond 6 the boundary is truncated. Then, the flight distance is again determined with the optical 7 property, $\beta$, in the next region. Throughout this study, it is assumed that the refractive 8 index is constant. If a particle escapes from the outer boundary, the particle is abandoned. 9 In that case, go to step (1), where the next new particle is emitted.

10 (3) Due to the second term on the right-hand side of Eq. (3), the weight changes 11 continuously as the particle flies because this term is not included in $\beta$, i.e., $\mu_{a}+\mu_{s}$. The 12 rate of weight change for a particle that flies an infinitesimal distance, $d s$, is described by:

$$
\frac{d \tilde{W}}{\tilde{W}}=-\frac{i \omega}{c} d s .
$$

14 After the particle flies a distance $s_{j}$ in the $j$ th flight path, the initial weight $\tilde{W}_{j}$ changes 15 to:

$16 \quad \tilde{W}_{j+1}=\tilde{W}_{j} \exp \left(-\frac{i \omega}{c} s_{j}\right)$,

17 which is obtained by solving the differential equation, Eq. (6). The method to include the 18 second term on the right-hand side of Eq. (3) is essentially the same as Eq. (3) in [19] that 19 is derived for spatial frequency domain calculations.

20 (4) At each collision site, both the real and imaginary parts of the complex-valued weight 21 are reduced from the probability of absorption:

$$
\tilde{W}_{j}^{\prime}=\tilde{W}_{j} \frac{\mu_{s}}{\beta}=\operatorname{Re}\left[\tilde{W}_{j}\right] \frac{\mu_{s}}{\beta}+i \cdot \operatorname{Im}\left[\tilde{W}_{j}\right] \frac{\mu_{s}}{\beta},
$$

23 where $\tilde{W}_{j}^{\prime}=$ the particle's weight after the weight reduction at the $(j-1)$ th collision site, $24 \operatorname{Re}[\cdot]=$ the real part, and $\operatorname{Im}[\cdot]=$ the imaginary part.

25 (5) Unless the particle leaves the outer boundary, the particle's random walk continues 26 endlessly while its weight reduces. If the medium is small enough compared to the mean 
1 free path of the medium, the particle leaves the outer boundary immediately. Otherwise, a 2 particle with a low weight should be killed by a Russian roulette method for the sake of 3 computational efficiency. The Russian roulette method is applied separately to the real and 4 imaginary parts when either or both of $\left|\operatorname{Re}\left[\tilde{W}_{j}^{\prime}\right]\right|$ and $\left|\operatorname{Im}\left[\tilde{W}_{j}^{\prime}\right]\right|$ are less than a 5 user-specified lower weight cutoff bound, $W_{L}$. In this study, the lower weight cutoff 6 bound is set to:

$$
W_{L}=C \cdot \max \left[\left|\operatorname{Re}\left[\tilde{W}_{s k}\right],\right| \operatorname{Im}\left[\tilde{W}_{s k}\right]\right],
$$

where $C$ is an input parameter that follows $0<C<1$, and $\tilde{W}_{s k}$ is the weight of the source particle given in the step (1). In the Russian roulette method, a new weight $C_{s u r} \cdot \operatorname{Re}\left[\tilde{W}_{j}^{\prime}\right]$ or $C_{\text {sur }} \cdot \operatorname{Im}\left[\tilde{W}_{j}^{\prime}\right]$ is given to the real or imaginary part of the surviving weight, respectively, if $\xi<1 / C_{\text {sur }}$ where $C_{\text {sur }}$ is a user-specified survival weight multiplier and $C_{s u r}>1$. Conversely, the real or imaginary part is killed if $\xi>1 / C_{\text {sur }}$. If either the real or imaginary part is killed, but the other part still survives, the particle is continually transported. The particle with a complex-valued weight is killed by the Russian roulette method only when both its real and imaginary parts are killed simultaneously. If the particle is killed by the Russian roulette method, go to step (1), and start the next new particle from the source point.

(6) A particle that survives the Russian roulette method is scattered in the direction that is sampled from the probability density function $f\left(\mathbf{r}, \mathbf{\Omega}^{\prime} \rightarrow \boldsymbol{\Omega}\right)$. The cosine of the angle $\theta$ between $\boldsymbol{\Omega}^{\prime}$ and $\boldsymbol{\Omega}$ is sampled [33]:

$$
\cos \theta=\frac{1}{2 g}\left(1+g^{2}-\left[\frac{1-g^{2}}{1-g+2 g \xi}\right]^{2}\right) .
$$

(7) When a particle with a weight $\tilde{W}$ leaves a boundary, the boundary measurement is

$$
\tilde{W} \cdot(\mathbf{n} \cdot \mathbf{\Omega}),
$$

where $\boldsymbol{\Omega}=$ particle's direction and $\mathbf{n}=$ the unit outward vector normal to the boundary surface, and the boundary is considered to be transparent and non-reflecting.

(8) Steps (1)-(7) are repeated until the desired statistics are obtained. The detector 
prediction at the boundary is estimated to be

$$
\tilde{P}_{d}=\frac{1}{N} \sum_{i} \tilde{W}_{i} \cdot\left(\mathbf{n} \cdot \mathbf{\Omega}_{i}\right)
$$

3 where $\tilde{W}_{i}=i$ th particle's weight leaving the boundary, and $i$ is summed over all particles

4 leaving the boundary, and $N=$ the total number of particles emitted from the source.

\section{Perturbation Monte Carlo}

To reconstruct the optical properties from the measurements, we need to estimate the sensitivity of the detector predictions $\tilde{P}_{d}$ or an objective function with respect to the optical properties $\boldsymbol{\mu}$ where $\boldsymbol{\mu}=\left[\mu_{a}(\mathbf{r}), \mu_{s}(\mathbf{r})\right]$. The adjoint differentiation technique has been commonly used for sensitivity or gradient estimation for the deterministic methods [2-13]. Although implementing the adjoint differentiation technique into the Monte Carlo method is not impossible, the perturbation Monte Carlo technique is more suitable, as demonstrated in previous work $[17,18,23,26-28]$. The perturbation Monte Carlo technique can provide the derivatives of the detector predictions with respect to the optical properties within every discretized domain by performing a single and forward Monte Carlo calculation. The increase in computation time caused by adding the perturbation calculation is very limited. There are two common techniques for perturbation Monte Carlo: the correlated sampling method and the Taylor series approach [34]. In the previous work on optical tomography, the correlated sampling method has been used. As shown in [34], the correlated sampling method always leads to a larger variance than the Taylor series approach. We wish to estimate the sensitivity of the detector predictions as accurately as possible with a limited computation time because each perturbed domain is small and we have to iterate the calculation many times. Therefore, this study chooses the first-order Taylor series approach (hereafter dubbed the "first-order differential operator method" [35]) to obtain the derivatives of the detector prediction with respect to the optical properties. Because we are interested in the first-derivative of the measurements with respect to the optical properties, we do not need to estimate higher order effects. Because 
the mathematical expression of the differential operator method is involved [36], we show

2 the brief essence used for this study. The probability density function of a free flight

3 distance $s$ (i.e., transport kernel) is $p(s)=\beta \exp (-\beta s)$ where $\beta=\mu_{a}+\mu_{s}$. The

4 first-derivative of the relative probability density function with respect to an absorption

$$
\begin{aligned}
& \frac{1}{C} \frac{\partial C}{\partial \mu_{a}}=\frac{1}{\mu_{s}} \frac{\partial \mu_{s}}{\partial \mu_{a}}-\frac{1}{\beta} \frac{\partial \beta}{\partial \mu_{a}}=-\frac{1}{\beta}, \\
& \frac{1}{C} \frac{\partial C}{\partial \mu_{s}}=\frac{1}{\mu_{s}} \frac{\partial \mu_{s}}{\partial \mu_{s}}-\frac{1}{\beta} \frac{\partial \beta}{\partial \mu_{s}}=\frac{1}{\mu_{s}}-\frac{1}{\beta},
\end{aligned}
$$

where $s$ is a flight distance within a perturbed region and we use $\partial \beta / \partial \mu_{a}=\partial \beta / \partial \mu_{a}=1$. If the particle passes through the perturbed region without stopping, the first term on the right-hand side in Eq. (13) or (14) is omitted. When the particle undergoes a collision in the perturbed region, the particle's weight changes by $C\left(=\mu_{s} / \beta\right)$ (i.e., collision kernel). The first-derivative of the relative collision kernel with respect to $\mu_{a}$ and $\mu_{s}$, respectively, is

where we use $\partial \mu_{s} / \partial \mu_{a}=0$. We combine Eqs. (13)-(16) to obtain the first-derivative of a detector prediction with respect to $\mu_{a, k}$ and $\mu_{s, k}$ in the region $k$, respectively:

$$
\begin{aligned}
& \frac{\partial \tilde{P}_{d}}{\partial \mu_{a, k}}=-\frac{1}{N} \sum_{i} \tilde{W}_{i} \cdot\left(\mathbf{n} \cdot \mathbf{\Omega}_{i}\right) S_{i, k}, \\
& \frac{\partial \tilde{P}_{d}}{\partial \mu_{s, k}}=\frac{1}{N} \sum_{i} \tilde{W}_{i} \cdot\left(\mathbf{n} \cdot \mathbf{\Omega}_{i}\right)\left(\frac{M_{i, k}}{\mu_{s, k}}-S_{i, k}\right),
\end{aligned}
$$

where $S_{i, k}=$ sum of the path lengths in the perturbed region $k$ for $i$ th detected particle, and $M_{i, k}=$ number of collisions in the perturbed region $k$ for $i$ th detected particle. $S_{i, k}$ and $M_{i, k}$ are accumulated during the course of the random walk for the forward Monte Carlo calculation with unperturbed optical properties.

For the purpose of reconstructing the optical properties, each time a particle flies or 
1 collides in each discretized image domain, the path length and the number of collisions in 2 that domain are recorded. When a particle is detected at the boundary, the derivative 3 defined by Eqs. (17) and (18) is scored as the derivative with respect to the optical 4 properties of the domain. Because one particle flies and collides in many domains before it 
1 calculated with the unperturbed or perturbed coefficient. Table 1 compares the differences 2 in the detector predictions obtained by direct subtraction and the perturbation method. The 3 perturbation in Table 1 is caused by the change in the absorption coefficient. For the 4 smaller perturbation (the upper part of Table 1), the relative differences between the 5 reference solution and the perturbation method fall within a few percent. The perturbation 6 method is approximately 3200 times faster than the direct subtraction method for the same 7 degree of statistical uncertainty. For the larger perturbation, the results of the perturbation method become much worse, as expected based on the properties of the first-order approximation. The relative differences are larger than $8 \%$.

Table 2 compares the results of the perturbation for the scattering coefficient. The computation efficiency of the perturbation method for the scattering coefficient is worse than for the absorption coefficient. This is because the two terms in parentheses in Eq. (18), $M_{i, k} / \mu_{s, k}$ and $S_{i, k}$, have very similar values, each of which has a statistical uncertainty. Thus, the statistical uncertainty of Eq. (18) is larger than that of Eq. (17). The perturbation method for the smaller perturbation is at most 30 times faster than the direct subtraction method, which is less effective compared to the perturbation for the absorption coefficient. However, the accuracy of the perturbation method for the scattering coefficient is comparable to the perturbation of the absorption coefficient. Consequently, it can be conjectured from the results for the smaller perturbation that the first-order derivative of the detector prediction, which is used for the reconstruction of the optical properties, can be accurately estimated by the first-order differential operator method.

[Fig. 1][Table 1][Table 2]

\section{Reconstruction Algorithm}

There have been many algorithms for reconstruction of optical properties from detector predictions. One method is to reconstruct the optical properties so that the objective function is minimized. The objective function is written as: 


$$
\phi=\frac{1}{2} \sum_{i=1}^{N_{d}}\left\|\tilde{P}_{d, i}-\tilde{M}_{d, i}\right\|^{2},
$$

where $\tilde{M}_{d, i}=$ measurement for $i$ th detector-source pair, and $N_{d}=$ total number of source-detector pairs. Another objective function, which is normalized to the

4 measurements, can be defined as:

$$
\phi=\frac{1}{2} \sum_{i=1}^{N_{d}}\left\|\frac{\tilde{P}_{d, i}-\tilde{M}_{d, i}}{\tilde{M}_{d, i}}\right\|^{2} .
$$

6 The minimization can be done by differentiating Eq. (19) or (20) with respect to the

unknown parameters. In many papers related to optical tomography [2-13], the derivative of $\phi$ with respect to the optical properties is calculated by solving the adjoint equation. As

9 shown in the previous section, the Monte Carlo method can easily obtain the derivative of

a detector prediction with respect to the optical properties in each discretized image domain. This paper does not choose a reconstruction method that minimizes the objective function. Instead, this paper chooses a reconstruction method that is used in Refs. [36, 37] as shown below.

The update vector for the optical properties, $\delta \boldsymbol{\mu}=\left[\delta \boldsymbol{\mu}_{a}, \delta \boldsymbol{\mu}_{s}\right]^{T}$, is determined from a linearized Newton-Raphson scheme:

$$
\Gamma=\mathbf{J} \delta \boldsymbol{\mu}
$$

The update vector is $\delta \boldsymbol{\mu}_{a}=\left[\delta \mu_{a, 1}, \delta \mu_{a, 2}, \cdots, \delta \mu_{a, K}\right]^{T}$ and $\delta \boldsymbol{\mu}_{s}=\left[\delta \mu_{s, 1}, \delta \mu_{s, 2}, \cdots, \delta \mu_{s, K}\right]^{T}$ where $K=$ total number of discretized image domains. It is assumed that each discretized image domain is homogeneous and that the optical properties are constant within the domain. $\boldsymbol{\Gamma}$ is an $N_{d}$-dimensional column vector whose elements are

$$
\tilde{\Gamma}_{i} \equiv \frac{\tilde{P}_{d, i}-\tilde{M}_{d, i}}{\tilde{M}_{d, i}}, \quad\left(i=1,2, \ldots, N_{d}\right) .
$$

$\mathbf{J}$ is a Jacobian matrix whose dimension is $N_{d} \times 2 K$. The $(i, j)$ element of $\mathbf{J}, J_{i, j}$, is

$$
\begin{aligned}
J_{i, j} & =\frac{1}{\tilde{M}_{d, i}} \frac{\partial \tilde{P}_{d, i}}{\partial \mu_{a, j}} \quad \text { for } 1 \leq j \leq K, \\
J_{i, j} & =\frac{1}{\tilde{M}_{d, i}} \frac{\partial \tilde{P}_{d, i}}{\partial \mu_{s, j}} \quad \text { for } K+1 \leq j \leq 2 K .
\end{aligned}
$$


1 At the beginning of a calculation, the detector predictions $\tilde{P}_{d, i}$ and the Jacobian matrix

$2 \mathbf{J}$ are calculated with an initial guess at the optical properties. To obtain the update vector 3 by solving Eq. (21), the algebraic reconstruction technique (ART), which is known to offer 4 a robust mode for large-scale, ill-posed linear systems, is used in this study [36, 37]. Once 5 we obtain the Jacobian matrix defined by Eqs. (23) and (24), the iterative step for the 6 update vector is

$$
\delta \boldsymbol{\mu}^{(j+1)}=\delta \boldsymbol{\mu}^{(j)}+\lambda \frac{\operatorname{Re}\left[\left(\tilde{\Gamma}_{j}-\mathbf{J}^{(j)} \cdot \delta \boldsymbol{\mu}^{(j)}\right) \cdot \mathbf{J}^{*(j) T}\right]}{\left\|\mathbf{J}^{(j)}\right\|^{2}}, \quad\left(j=0,1, \ldots, N_{d}\right),
$$

8 where the asterisk denotes a complex conjugation, $\mathbf{J}^{(j)}=j$ th row of $\mathbf{J}, \delta \boldsymbol{\mu}^{(0)}=\mathbf{0}$, and $9 \lambda=$ relaxation parameter in the interval $(0,2)$. This iteration procedure constitutes the inner 10 iteration for the update vector. Then, the updated optical properties for the next (outer) 11 iteration are

$$
\boldsymbol{\mu}_{k+1}=\boldsymbol{\mu}_{k}+\delta \boldsymbol{\mu}_{k},
$$

where the subscript $k$ denotes the $k$ th outer iteration. Using the updated optical properties, new detector predictions $\tilde{P}_{d, i}$ and a new Jacobian matrix $\mathbf{J}$ are calculated.

The objective function $\phi$ defined in Eq. (19) or (20) is used as a criterion to terminate the outer iteration. Due to the stochastic nature of the Monte Carlo method, the objective function $\phi$ would no longer decrease below a certain level and $\phi$ shifts to a fluctuation mode, even if the outer iteration is repeated. Unlike deterministic methods, the convergence of the Monte Carlo method for optical tomography is not clearly identified. As an alternate convergence criterion for the Monte Carlo method, the outer iteration is terminated when the objective function does not fall to a new low for as many iteration times as prescribed. To avoid a premature termination due to an abnormally small value of the objective function, a minimum number of outer iterations must be performed.

\section{Numerical Examples}

\subsection{Description of numerical tests}

The geometry and the background coefficients are the same as those used in Sec. 3 . 
1 That is, $\mu_{a}=0.5 \mathrm{~cm}^{-1}$ and $\mu_{s}=50 \mathrm{~cm}^{-1}$. As shown in Fig. 2, the $2 \mathrm{~cm} \times 2 \mathrm{~cm}$ domain is 2 discretized into a $41 \times 41$ square domain. Each discretized domain is considered 3 homogeneous and has its own optical properties. Four zero-phased point sources with a 4 modulation frequency of $600 \mathrm{MHz}$ are positioned at the center of every side. Each side is 5 equally divided into 9 pieces, each of which is used as a detector region with a length of $62 / 9 \mathrm{~cm}$. Thus, the total number of the source-detector pairs is $4 \times 4 \times 9=144$. The anisotropy factor is $g=0.9$. From each source position, $1 \times 10^{6}$ particles are emitted into the medium per outer iteration, which means a total of $4 \times 10^{6}$ particles are spent in the outer iteration. The average computation time per outer iteration is approximately 4 min on a PC with $3.33 \mathrm{GHz} \mathrm{CPU}$. The initial values for the optical properties are set to be the same as the background. Before starting the iterative procedures for optical tomography, a light transport calculation is performed with real optical properties and a sufficiently large computation time. The result of this calculation is used as a "measured" value, $\tilde{M}_{d, i}$. Although a regularization technique for ART is proposed in [38], this study performs the reconstruction of optical properties without it to investigate how the proposed method works for an ill-posed inverse problem without the regularization technique.

We handle four inverse problems: (1) single inclusion and retrieval of the absorption coefficient only, (2) single inclusion and simultaneous retrieval of both absorption and scattering coefficients, (3) double inclusion and retrieval of the absorption coefficient only, and (4) double inclusion and simultaneous retrieval of the absorption and scattering coefficients. Cases (1) and (3) are intended to show the feasibility of the proposed method for optical tomography of a less ill-posed problem. Cases (2) and (4) are performed for the purpose of testing the robustness of the proposed method in the presence of cross-talk between absorption and scattering coefficients.

[Fig. 2]

$26 \quad 5.2$ Single inclusion and retrieval of absorption coefficient only

27 The distribution of the absorption coefficient is shown in Fig. 3 (a). The absorption 
1 coefficient for the inclusion is $\mu_{a}=0.8 \mathrm{~cm}^{-1}$, while the background has coefficient

$2 \mu_{a}=0.5 \mathrm{~cm}^{-1}$. The reconstructed distribution of the absorption coefficient is shown in Fig.

33 (b). Fig. 4 shows a comparison of the real and reconstructed absorption coefficients along

4 a diagonal line from the bottom-left corner to the top-right corner. In this calculation, the

5 relaxation parameter defined in Eq. (25) is set to $\lambda=0.15$. The larger $\lambda$ leads to faster

6 convergence, but the reconstructed distribution is unstable. On the other hand, although the

7 smaller $\lambda$ leads to a stable distribution, the convergence per iteration is slow. The

8 relaxation parameter $\lambda=0.15$ is empirically chosen as an optimal parameter. Fig. 5 shows

9 the objective function defined in Eq. (20) plotted against the outer iteration. The iteration is 10 terminated when the objective function does not update a new low for 10 iterations. The 11 convergence speed is relatively low after the 10th iteration. As seen from Figs. 3 and 4, a 12 single inclusion with a higher absorption coefficient is well identified using the algorithm 13 for the proposed method.

155.3 Single inclusion and simultaneous retrieval of absorption and scattering coefficients 16 In this example, a single inclusion has optical properties $\mu_{a}=0.8 \mathrm{~cm}^{-1}$ and $\mu_{s}=70$ $17 \mathrm{~cm}^{-1}$, which are higher than the background. The distributions of the real optical properties 18 are shown in Figs. 6(a) and (b). As discussed in Sec. 3, the first-derivative of a detector 19 prediction with respect to the scattering coefficient is less sensitive and has more statistical 20 noise compared to an absorption coefficient. For this reason, the convergence of the 21 scattering coefficients is slower and the reconstructed map of the scattering coefficients is 22 not as clear as the map for the absorption coefficient. Fig. 6 shows the real and 23 reconstructed optical properties. Despite the cross-talk between the absorption and 24 scattering coefficients, the distributions of higher absorption and scattering coefficients are 25 well distinguished, although some undershooting in the reconstructed scattering 26 coefficients is observed around the inclusion. Because the convergence speed of the 27 scattering and absorption coefficients are mismatched, different relaxation parameters $\lambda$ in 
Eq. (25) are assigned to scattering and absorption coefficients. The relaxation parameters for scattering and absorption coefficients are 0.18 and 0.10 , respectively. Figs. 7 (a) and (b) show the comparison of the real and reconstructed scattering and absorption coefficients, respectively, along a diagonal line from the bottom-left corner to the top-right corner. While the absorption coefficient is well reconstructed, the scattering coefficient shows a relatively unstable distribution. The cross-section view of the reconstructed optical properties is also published in $[4,5,12]$. The proposed Monte Carlo method can provide well-reconstructed distributions that are comparable to the results of the deterministic approach.

[Fig. 6], [Fig. 7]

5.4 Double inclusions and retrieval of the absorption coefficient alone

In this example, another inclusion with $\mu_{a}=0.2 \mathrm{~cm}^{-1}$ is added to the example in Sec. 5.2. The scattering coefficient is fixed at $\mu_{s}=50 \mathrm{~cm}^{-1}$ throughout the medium. The real and reconstructed absorption coefficients are shown in Figs. 8 (a) and (b), respectively. Fig. 9 shows the real and reconstructed absorption coefficients along a diagonal line from the bottom-left corner to the top-right corner. Although the absolute value of the absorption coefficient is not fully reproduced, the shapes and locations of the inclusions are well reconstructed using the proposed Monte Carlo algorithm.

[Fig. 8], [Fig. 9]

5.5 Double inclusions and simultaneous retrieval of scattering and absorption coefficients The last example investigates the robustness of the proposed Monte Carlo method for a cross-talk problem. One inclusion is composed of a denser material: $\mu_{a}=0.8 \mathrm{~cm}^{-1}$ and $\mu_{s}=70 \mathrm{~cm}^{-1}$. Another inclusion is composed of a more transparent material: $\mu_{a}=0.2$ $\mathrm{cm}^{-1}$ and $\mu_{s}=30 \mathrm{~cm}^{-1}$. The relaxation parameters $\lambda$ for the scattering and absorption coefficients are 0.18 and 0.10 , respectively, which are the same as in Sec. 5.3 for the single inclusion problem. Fig. 10 shows the real and reconstructed optical properties. Figs. 11 (a) and (b) show the comparison of the real and reconstructed optical properties along 


\begin{abstract}
1 a diagonal line from the bottom-left corner to the top-right corner. Fig. 12 shows the 2 objective function defined in Eq. (20) versus the outer iteration. As seen from Figs. 10 3 and 11, the locations of the two inclusions are well identified. The reconstructed 4 absorption coefficient is somewhat unstable compared to the results in Sec. 5.4 because 5 the simultaneous reconstruction of optical properties is ill-posed. The reconstruction of 6 the absorption coefficient is better than that of the scattering coefficient, as shown in Sec. 7 5.3. Again, the quality of the reconstructed results for the proposed Monte Carlo method 11 reasonable computational resources. approximately 40 times, and each cycle takes approximately 4 minutes. Thus, in terms of computational efficiency, the proposed Monte Carlo method can be performed with
\end{abstract}

[Fig. 10], [Fig. 11], [Fig. 12]

\title{
5.6 Comparison with the results of a deterministic method
}

The accuracy and efficiency of the present Monte Carlo method are compared with the results of a deterministic method that is recently published in [13]. A Monte Carlo calculation is performed for the case whose results are shown in Fig. 9 in [13]. A two-dimensional homogeneous $4 \mathrm{~cm} \times 4 \mathrm{~cm}$ domain is used in [13]. The medium contains two $1 \mathrm{~cm} \times 1 \mathrm{~cm}$ square inclusions. The absorption and scattering coefficients are $0.5 \mathrm{~cm}^{-1}$ and $1.0 \mathrm{~cm}^{-1}$ in the background, respectively, and the anisotropic factor is $g$ $=0.8$. A steady-state collimated source is positioned at the center of every side (i.e., $\omega=0$ in Eq. (3)), and 16 detectors are allocated evenly over the outer surface. The domain is discretized into a $20 \times 20$ square domain, which is almost the same resolution as in [13]. The optical parameters of the inclusions are $\mu_{a}=0.1 \mathrm{~cm}^{-1}$ and $\mu_{s}=0.5 \mathrm{~cm}^{-1}$ for one inclusion, and $\mu_{a}=1.0 \mathrm{~cm}^{-1}$ and $\mu_{s}=1.5 \mathrm{~cm}^{-1}$ for another inclusion.

Fig. 13 shows the real and reconstructed optical properties obtained by the Monte Carlo method. For comparison with the results in the deterministic method, Fig. 13 is shown with the same image style as in [13]. Again, the locations of the two inclusions are 
1 well identified. As a quantitative index of the reconstruction, the normalized root-mean 2 square error (NRMSE) is introduced in [13] and defined as

$$
\text { NRMSE }=\left[\frac{\sum_{i=1}^{K}\left(\hat{\mu}_{i}-\tilde{\mu}_{i}\right)^{2}}{\sum_{i=1}^{K} \tilde{\mu}_{i}^{2}}\right]^{1 / 2},
$$

4 where $K=$ total number of discretized image domains, $\tilde{\mu}_{i}=$ real optical property, and $5 \hat{\mu}_{i}=$ reconstructed optical property. Table 3 compares the NRMSE of the deterministic 6 method in Table 1 in [13] with the Monte Carlo method of the present paper. While the 7 Monte Carlo method is a little worse for the absorption coefficient, it is better for the 8 scattering coefficient. Although it cannot be concluded which method is superior, the 9 Monte Carlo method is comparable to the deterministic method in [13] in terms of the 10 NRMSE. According to [13], the computation time for one iteration is 20 minutes on a PC 11 with $3.4 \mathrm{GHz}$ CPU. The total iteration may be more than 50 times. On the other hand, the 12 Monte Carlo method spends approximately 2 minutes for one iteration on the same level $13 \mathrm{CPU}(3.33 \mathrm{GHz})$, and the total iteration is 74 times. In terms of the computational 14 efficiency, the Monte Carlo method can be a viable method for the optical tomography.

\section{Conclusions}

Near-infrared optical tomography that utilizes the Monte Carlo technique has been developed by many researchers. In optical tomography, an intensity-modulated light source with a given modulation frequency is a very useful approach because the use of frequency data can provide a better separation between the absorbing and scattering objects. Thus far, some previous work has been performed on problems of optical tomography using the Monte Carlo method. This paper presents a new study of the reconstruction of optical properties for a heterogeneous, two-dimensional tissue model in the frequency domain.

In optical tomography that uses deterministic methods, the derivative of the objective 
1 function or detector prediction (i.e., Jacobian matrix) is obtained by the adjoint 2 differentiation scheme. On the other hand, the Monte Carlo method can easily yield the 3 derivative using a perturbation Monte Carlo technique. In previous Monte Carlo studies for 4 optical tomography, the derivative has been obtained by the so-called "correlated 5 sampling" method. This study introduces another Monte Carlo perturbation technique, the 6 first-order differential operator method, which is expected to give smaller variances. In Sec. 73 , it is shown that the differential operator technique can accurately yield the 8 first-derivative of the detector prediction with respect to optical parameters such as the 9 absorption coefficient and the scattering coefficient.

10 In most deterministic optical tomography, the optical properties are reconstructed to 11 minimize the objective function. The objective function is usually the mean-squared norm 12 of the relative difference between the measured data and the detector prediction. Due to the 13 stochastic nature of the Monte Carlo method, this procedure does not always provide a 14 continuously stable solution for optical tomography in a two-dimensional heterogeneous 15 medium, according to our experiences in this study. In this study, the update vector for the 16 optical properties is determined from a linearized Newton-Rapson scheme. To obtain the update vector, the algebraic reconstruction technique (ART) is used.

The proposed Monte Carlo algorithm is successfully applied to achieve simultaneous reconstruction of the scattering and absorption coefficients. The quality of the reconstructed results compares favorably with the deterministic or Monte Carlo methods performed thus far. The computational efficiency of the proposed method is equivalent or superior to the existing deterministic or Monte Carlo methods. It would be possible to further improve the speed by incorporating a parallel computing technique. Although a square grid is used for the discretized image domain in this study, any arbitrary shape is possible for individual small domains, such as a triangular element in the finite element method. Further studies will focus on exploring the possibility minimizing the objective function using its derivative. This procedure, which was not adopted in this study, would 
2

further expand the possibilities for optical tomography using the Monte Carlo method.

At the moment, the authors cannot make any mention about whether the Monte Carlo

3 method presented in this paper works well for the optical tomography in three-dimensional

4 geometries. Due to the superior flexibility of the Monte Carlo method for geometry

5 handling, the expansion to general three-dimensional geometries would be an interesting

6 issue for future work.

\section{$8 \quad$ References}

9 [1] A.H. Hielscher, R.E. Alcouffe, R.L. Barbour, Comparison of finite-difference 10 transport and diffusion calculations for photon migration in homogeneous and heterogeneous tissues, Phys. Med. Biol. 43 (1998) 1285-1302.

[2] S.R. Arridge, J.C. Hebden, Optical imaging in medicine: II Modelling and reconstruction, Phys. Med. Biol. 42 (1997) 841-853.

[3] A.D. Klose, U. Netz, J. Beuthan, A.H. Hielscher, Optical tomography using the time-independent equation of radiative transfer - Part 1: forward model, J. Quant. Spectrosc. Radiat. Transf. 72 (2002) 691-713.

[4] A.D. Klose, A.H. Hielscher, Optical tomography using the time-independent equation of radiative transfer - Part 2: inverse model, J. Quant. Spectrosc. Radiat. Transf. 72 (2002) 715-732.

[4] K. Ren, G. Bal, A.H. Hielscher, Frequency domain optical tomography based on the equation of radiative transfer, SIAM J. Sci, Comput. 28, No.4 (2006) 1463-1489.

[5] H.K. Kim, A. Charette, A sensitivity function-based conjugate gradient method for optical tomography with the frequency-domain equation of radiative transfer, J. Quant. Spectrosc. Radiat. Transf. 104 (2007) 24-39.

[6] H.K. Kim, A. Charette, Frequency domain optical tomography using a conjugate gradient method without line search, J. Quant. Spectrosc. Radiat. Transf. 104 (2007) $248-256$. 
[7] A. Charette, J. Boulanger, H.K. Kim, An overview on recent radiation transport algorithm development for optical tomography imaging, J. Quant. Spectrosc. Radiat. Transf. 109 (2008) 2743-2766.

[8] T. Tarvainen, M. Vauhkonen, S.R. Arridge, Gauss-Newton reconstruction method for optical tomography using the finite element solution of the radiative transfer equation, $\mathrm{J}$. Quant. Spectrosc. Radiat. Transf. 109 (2008) 2767-2778.

[9] O. Balima, T. Pierre, A. Charette, D. Marceau, A least square finite element formulation of the collimated irradiation in frequency domain for optical tomography applications, J. Quant. Spectrosc. Radiat. Transf. 111 (2010) 280-286.

[10] O. Balima, J. Boulanger, A. Charette, D. Marceau, New development in frequency domain optical tomography. Part I: Forward model and gradient computation, J. Quant. Spectrosc. Radiat. Transf. 112 (2011) 1229-1234.

[11] O. Balima, Y. Favennec, J. Boulanger, A. Charette, Optical tomography with the discontinuous Galerkin formulation of the radiative transfer equation in frequency domain, J. Quant. Spectrosc. Radiat. Transf. 112 (2012) 805-814.

[12] O. Balima, Y. Favennec, D. Rousse, Optical tomography reconstruction algorithm with the finite element method: An optimal approach with regularization tools, J. Compt. Phys. 251 (2013) 461-479.

[13] Y. Qiao, H. Qi, Q. Chen, L. Ruan, H. Tan, Multi-start iterative reconstruction of the radiative parameter distributions in participating media based on the transient radiative transfer equation, Opt. Commun. 351 (2015) 75-84.

[14] T.O. McBride, B.W. Pogue, U. L. Österberg, K. D. Paulsen, Separation of absorption and scattering heterogeneities in NIR tomographic imaging of tissue, in OSA Technical Digest, Biomedical Topical Meetings, Optical Society of America, Washington, DC, (2000).

[15] C. Zhu, Q. Li, Review of Monte Carlo modelling of light transport in tissues, J. Biomed. Opt. 18(5) (2013) 050902. 
[16] M. Testorf, U. Österberg, B. Pogue, K. Paulsen, Sampling of time- and frequency-domain signals in Monte Carlo simulations of photon migration, Appl. Opt. 38(1) (1999) 236-245.

[17] C.K. Hayakawa, J. Spanier, F. Bevilacqua, A.K. Dunn, J.S. You, B.J. Tromberg, V. Venugopalan, Perturbation Monte Carlo methods to solve inverse photon migration problems in heterogeneous tissues, Opt. Lett. 26(17) (2001) 1335-1337.

[18] A. Sassaroli, Fast perturbation Monte Carlo method for photon migration in heterogeneous turbid media, Opt. Lett. 36(11) (2011) 2095-2097.

[19] A.R. Gardner, V. Venugopalan, Accurate and efficient Monte Carlo solutions to the radiative transport equation in the spatial frequency domain, Opt. Lett. 36(12) (2011) 2269-2271.

[20] M. Francoeur, R. Vaillon, D.R. Rousse, Theoretical analysis of frequency and time-domain methods for optical characterization of absorbing and scattering media, $\mathrm{J}$. Quant. Spectrosc. Radiat. Transf. 93 (2005) 139-150.

[21] M. Francoeur, D.R. Rousse, Short-pulsed laser transport in absorbing and scattering media: time-based versus frequency-based approaches. J. Phys. D: Appl. Phys. 40 (2007) 5733-5742.

[22] Yamamoto, T., Sakamoto, H., Dynamic Monte Carlo calculation method by solving frequency domain transport equation using the complex-valued weight Monte Carlo method (in press).

[23] I. Seo, J.S. You, C.K. Hayakawa, V. Venugopalan, Perturbation and differential Monte Carlo methods for measurement of optical properties in a layered epithelial tissue model, J. Biomed. Opt. 12(1) (2007) 014030.

[24] H. Zhao, S. Zhang, Z. Wang, H. Miao, Z. Du, J. Jiang, Frequency-domain inverse Monte Carlo simulation for the diagnosis of the early cervical cancer based on NIR diffuse measurement, Progress in Biomedical Optics and Imaging - Proceedings of SPIE, Vol. 6859, 2008 (2008) Article number 68591N. 
[25] H. Zhao, X. Zhou, H. Li, J. Ma, K. Xu, Fast inverse Monte Carlo simulation for extracting the optical properties of cylindrical tissue, Progress in Biomedical Optics and Imaging - Proceedings of SPIE, Vol. 7174, 2008 (2009) Article number 71741P.

[26] M. Sharma, R. Hennessy, M.K. Markey, J.W. Tunnell, Verification of a two-layer inverse Monte Carlo absorption model using multiple source-detector separation diffuse reflectance spectroscopy, Biomed. Opt. Express 5(1) (2014) 40-53.

[27] Y.P. Kumar, R.M. Vasu, Reconstruction of optical properties of low-scattering tissue using derivative estimated through perturbation Monte-Carlo method, J. Biomed. Opt. 9(5) (2004) 1002-1012.

[28] P.K. Yalavarthy, K. Karlekar, H.S. Patel, R.M. Vasu, M. Pramanik, P.C. Mathias, B. Jain, P. K. Gupta, Experimental investigation of perturbation Monte-Carlo based derivative estimation for imaging low-scattering tissue, Opt. Express 13(3) (2005) 985-997.

[29] T. Yamamoto, Monte Carlo method with complex weights for neutron leakage-corrected calculations and anisotropic diffusion coefficient generations, Ann. Nucl. Energy 50 (2012) 141-149.

[30] T. Yamamoto, Monte Carlo method with complex-valued weights for frequency domain analyses of neutron noise, Ann. Nucl. Energy 58 (2013) 72-79.

[31] T. Yamamoto, Frequency domain Monte Carlo simulation method for cross power spectral density driven by periodically pulsed spallation neutron source using complex-valued weight Monte Carlo method. Ann. Nucl. Energy 63 (2014) 711-720.

[32] T. Yamamoto, H. Sakamoto, A new concept of Monte Carlo kinetics parameter calculation using complex-valued perturbation, Ann. Nucl. Energy 71 (2014) 480-488.

[33] S.A. Prahl, M. Kaijzer, S.L. Jacques, A.J. Welch, A Monte Carlo model of light propagation in tissue, SPIE Institute Series Vol. IS 5 (1989) 102-111.

[34] H. Rief, Generalized Monte Carlo perturbation algorithms for correlated sampling and a second-order Taylor series approach, Ann. Nucl. Energy 9 (1984) 455-476. 
[35] Y. Nagaya, T. Mori, Impact of perturbed fission source on the effective multiplication factor in Monte Carlo perturbation calculations, J. Nucl. Sci. Technol. 42(5) (2005) $428-441$.

[36] F. Gao, H. Zhao, Y. Tanikawa, Y. Yamada, Time-resolved diffuse optical tomography using a modified generalized pulse spectrum technique, IEICE Trans. Info. Sys. Vol. 85-D No. 1 (2002) 133-142.

[37] D. Gordon, Parallel ART for image reconstruction in CT using processor arrays, Int. J. Parallel Emergent Distrib. Syst. 21(5) (2006) 365-380.

9 [38] P.P.B. Eggermont, G.T. Herman, A. Lent, Iterative algorithms for large partitioned 10 linear systems, with applications to image reconstruction, Linear Algebra and its $11 \quad$ Applications 40 (1981) 37-67. 
Figure captions

2 Fig. 1 Geometry of test calculations for perturbation Monte Carlo.

3 Fig. $241 \times 41$ square grid, 4 source and 36 detector positions for Monte Carlo $4 \quad$ calculations.

5 Fig. 3 Distribution of absorption coefficient. (a) real distribution. (b) reconstructed 6 distribution (single inclusion).

7 Fig. 4 Real and reconstructed distributions of absorption coefficient along the diagonal

9 Fig.5 Objective function versus outer iteration for single inclusion and retrieval of $10 \quad$ absorption coefficient only.

11 Fig. 6 Distribution of scattering and absorption coefficients. (a), (b): real distributions. 12 (c), (d): reconstructed distributions.

13 Fig. 7 Real and reconstructed distributions of scattering coefficient (a) and absorption 14 coefficient (b) along the diagonal line from the bottom-left corner to the top-right 15 corner (case for single inclusion).

16 Fig. 8 Distribution of absorption coefficient. (a) real distribution. (b) reconstructed distribution.

Fig. 9 Real and reconstructed distributions of absorption coefficient along the diagonal line from the bottom-left corner to the top-right corner (case for double inclusions).

Fig. 10 Distribution of scattering and absorption coefficients. (a), (b): real distributions. (c), (d) reconstructed distributions (case for double inclusions).

Fig. 11 Real and reconstructed distributions of scattering coefficient (a) and absorption coefficient (b) along the diagonal line from the bottom-left corner to the top-right corner (case for double inclusions).

Fig.12 Objective function versus outer iteration for double inclusions and simultaneous retrieval of scattering and absorption coefficients. 

Fig. 13 Distribution of scattering and absorption coefficients. (a), (b): real distributions. (c),
2
(d) reconstructed distributions (case for Fig. 9 in [13]). 
Table 1 Difference of the detector predictions on the boundaries caused by the perturbation of absorption coefficient. Comparison of the direct calculations and perturbation calculations.

\begin{tabular}{|c|c|c|c|c|c|c|c|}
\hline $\begin{array}{c}\delta \mu_{a} \\
\left(\mathrm{~cm}^{-1}\right)\end{array}$ & & \multicolumn{2}{|c|}{ Real part of $\Delta \tilde{P}_{d}$} & \multirow[t]{2}{*}{$\begin{array}{l}\text { Relative } \\
\text { difference } \\
(\%)\end{array}$} & \multicolumn{2}{|c|}{ Imaginary part of $\Delta \tilde{P}_{d}$} & \multirow[t]{2}{*}{$\begin{array}{l}\text { Relative } \\
\text { difference } \\
(\%)\end{array}$} \\
\hline \multirow[t]{5}{*}{0.01} & & Reference & Perturbation & & Reference & Perturbation & \\
\hline & Side 1 & $-4.93 E-5^{a}$ & $-5.08 \mathrm{E}-5$ & 3.2 & 4.09E-5 & $4.23 \mathrm{E}-5$ & 3.3 \\
\hline & Side 2 & $-1.61 \mathrm{E}-4$ & $-1.64 \mathrm{E}-4$ & 1.8 & $9.00 \mathrm{E}-5$ & $9.19 \mathrm{E}-5$ & 2.1 \\
\hline & Side 3 & $-2.15 \mathrm{E}-4$ & $-2.12 \mathrm{E}-4$ & -1.7 & $1.02 \mathrm{E}-4$ & $1.03 \mathrm{E}-4$ & 1.6 \\
\hline & Side 4 & $-9.89 E-5$ & $-9.95 \mathrm{E}-5$ & 0.6 & $5.90 \mathrm{E}-5$ & $5.99 \mathrm{E}-5$ & 1.6 \\
\hline \multirow[t]{4}{*}{0.1} & Side 1 & $-4.18 \mathrm{E}-4$ & $-5.08 \mathrm{E}-4$ & 22 & $3.37 \mathrm{E}-4$ & $4.23 \mathrm{E}-4$ & 26 \\
\hline & Side 2 & $-1.45 \mathrm{E}-3$ & $-1.64 \mathrm{E}-3$ & 13 & $7.79 \mathrm{E}-4$ & $9.19 \mathrm{E}-4$ & 18 \\
\hline & Side 3 & $-1.97 \mathrm{E}-3$ & $-2.12 E-3$ & 7.5 & $9.27 \mathrm{E}-4$ & $1.03 \mathrm{E}-3$ & 11 \\
\hline & Side 4 & $-9.03 E-4$ & $-9.95 \mathrm{E}-4$ & 10 & $5.24 \mathrm{E}-4$ & $6.00 \mathrm{E}-4$ & 14 \\
\hline
\end{tabular}

${ }^{\mathrm{a}} \mathrm{read}$ as $-4.93 \times 10^{-5}$ 
Table 2 Difference of the detector predictions on the boundaries caused by the perturbation of scattering coefficient. Comparison of the direct calculations and perturbation calculations.

\begin{tabular}{|c|c|c|c|c|c|c|c|}
\hline $\begin{array}{c}\delta \mu_{s} \\
\left(\mathrm{~cm}^{-1}\right)\end{array}$ & & \multicolumn{2}{|c|}{ Real part of $\Delta \tilde{P}_{d}$} & \multirow[t]{2}{*}{$\begin{array}{l}\text { Relative } \\
\text { difference } \\
(\%)\end{array}$} & \multicolumn{2}{|c|}{ Imaginary part of $\Delta \widetilde{P}_{d}$} & \multirow[t]{2}{*}{$\begin{array}{l}\text { Relative } \\
\text { difference } \\
(\%)\end{array}$} \\
\hline \multirow[t]{5}{*}{0.8} & & Reference & Perturbation & & Reference & Perturbation & \\
\hline & Side 1 & $-4.92 \mathrm{E}-5^{\mathrm{a}}$ & $-5.09 \mathrm{E}-5$ & 3.3 & $2.82 \mathrm{E}-5$ & $2.90 \mathrm{E}-5$ & 2.9 \\
\hline & Side 2 & $-1.30 \mathrm{E}-4$ & $-1.32 \mathrm{E}-4$ & 2.0 & 4.59E-5 & $4.65 \mathrm{E}-5$ & 1.4 \\
\hline & Side 3 & $2.60 \mathrm{E}-4$ & $2.48 \mathrm{E}-4$ & -4.6 & $-7.09 \mathrm{E}-5$ & $-7.14 \mathrm{E}-5$ & 0.6 \\
\hline & Side 4 & $-5.79 \mathrm{E}-5$ & $-5.68 \mathrm{E}-5$ & -1.9 & $2.01 \mathrm{E}-5$ & $1.99 \mathrm{E}-5$ & -1.0 \\
\hline \multirow[t]{4}{*}{8.0} & Side 1 & $-4.14 \mathrm{E}-4$ & $-5.09 \mathrm{E}-4$ & 23 & $2.45 \mathrm{E}-4$ & $2.91 \mathrm{E}-4$ & 19 \\
\hline & Side 2 & $-1.16 \mathrm{E}-3$ & $-1.32 \mathrm{E}-3$ & 14 & $4.16 \mathrm{E}-4$ & $4.65 \mathrm{E}-4$ & 12 \\
\hline & Side 3 & $2.31 \mathrm{E}-3$ & $2.48 \mathrm{E}-3$ & 7.0 & $-6.61 \mathrm{E}-4$ & $-7.13 \mathrm{E}-4$ & 7.8 \\
\hline & Side 4 & $-5.28 \mathrm{E}-4$ & $-5.68 \mathrm{E}-4$ & 7.5 & $1.89 \mathrm{E}-4$ & $1.98 \mathrm{E}-4$ & 4.9 \\
\hline
\end{tabular}

${ }^{\mathrm{a}}$ read as $-4.92 \times 10^{-5}$ 
Table 3 Normalized root-mean square errors by the deterministic method and the Monte Carlo method.

\begin{tabular}{lcc}
\hline & Absorption & Scattering \\
\hline \multicolumn{3}{c}{ NRMSE } \\
\hline Deterministic (Ref. [13]) & 0.131 & 0.0935 \\
\hline Monte Carlo & 0.144 & 0.0932 \\
\hline
\end{tabular}




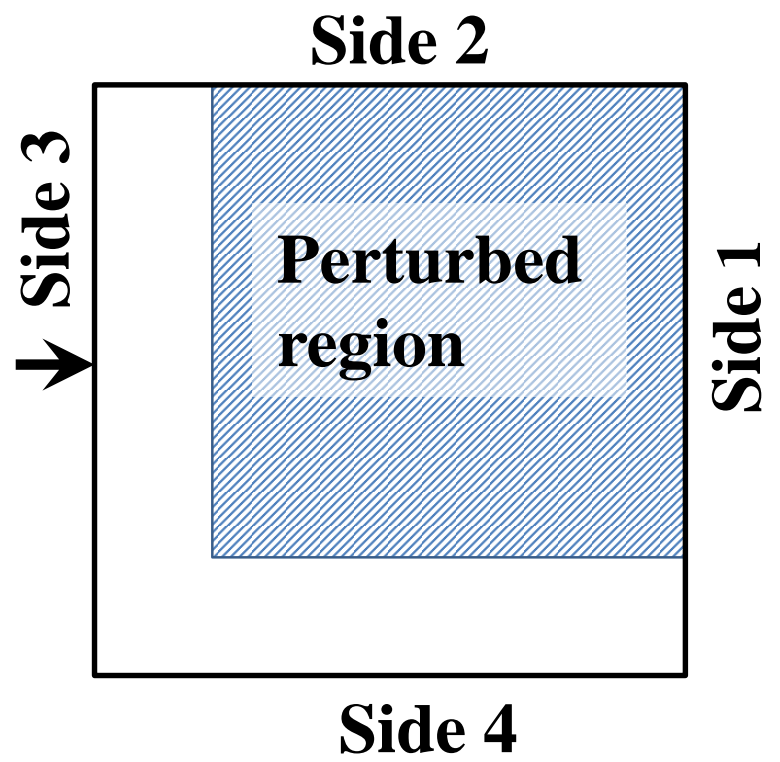

Fig. 1 Geometry of test calculations for perturbation Monte Carlo. 


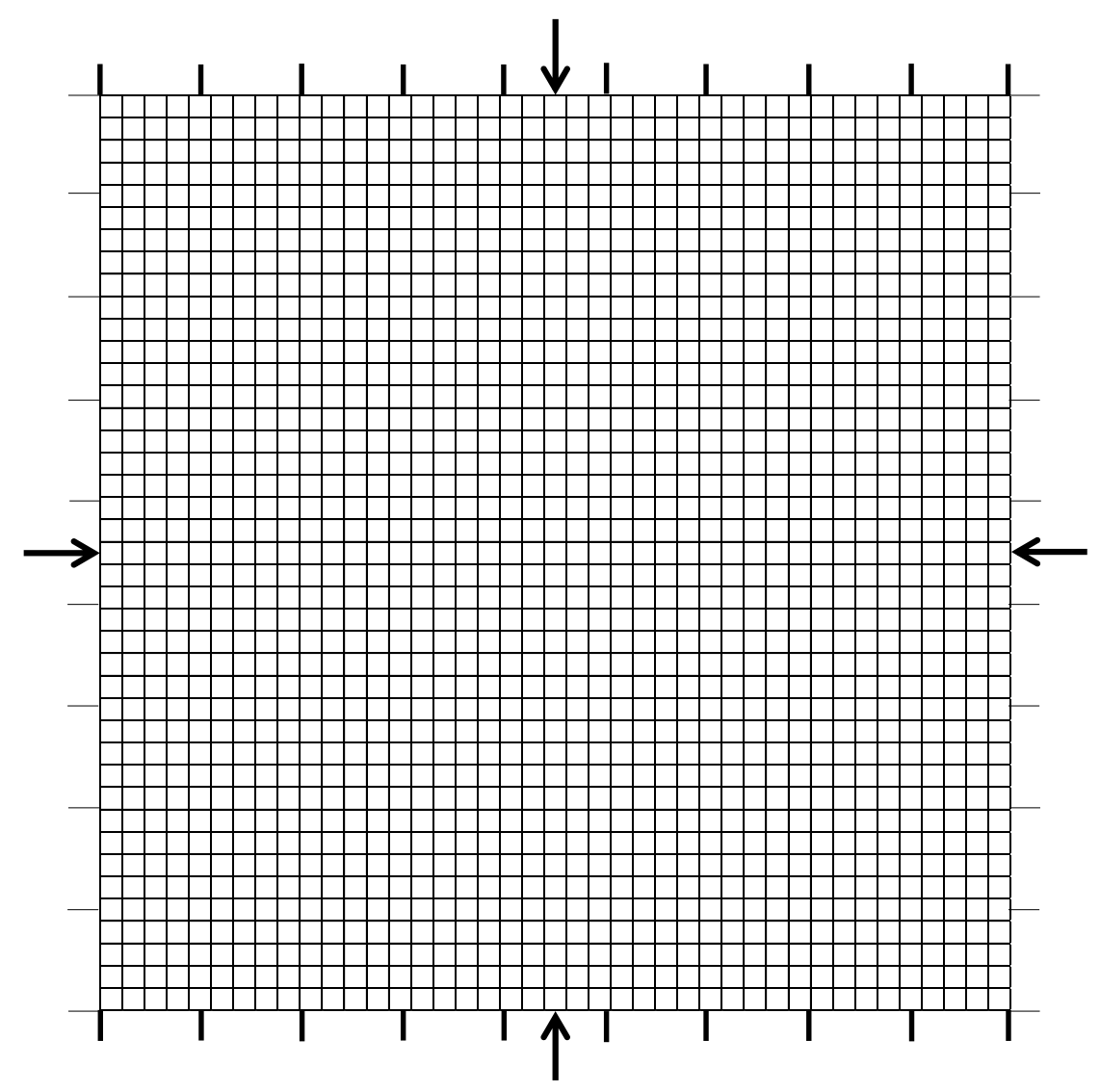

Fig. $241 \times 41$ square grid, 4 source and 36 detector positions for Monte Carlo calculations.

\section{T. Yamamoto}




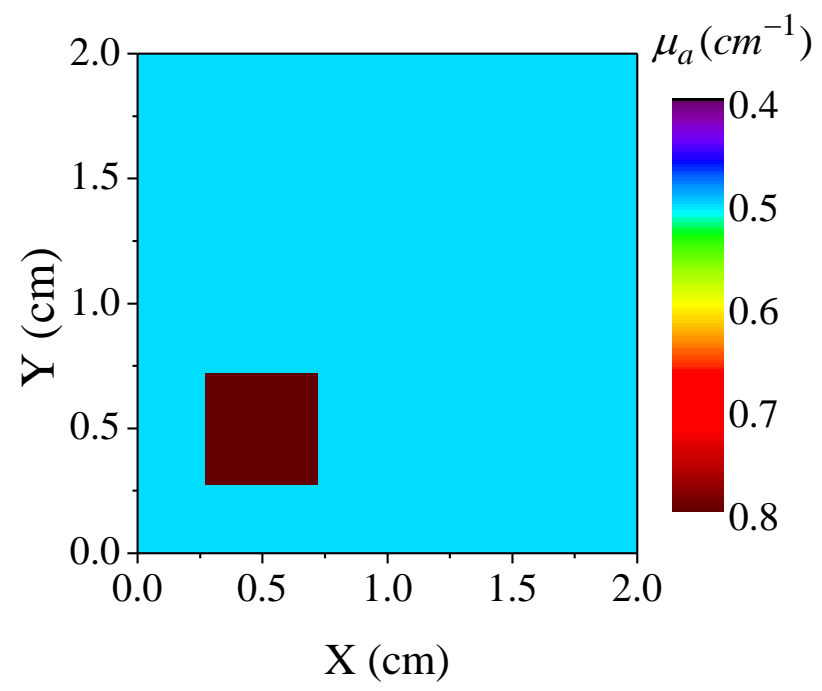

(a)

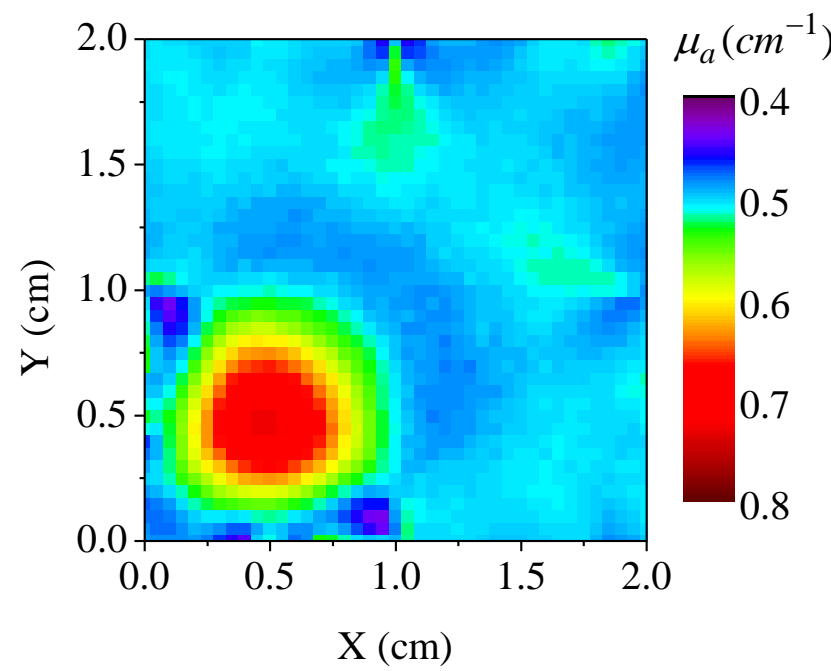

(b)

Fig. 3 Distribution of absorption coefficient. (a) real distribution. (b) reconstructed distribution (single inclusion). 


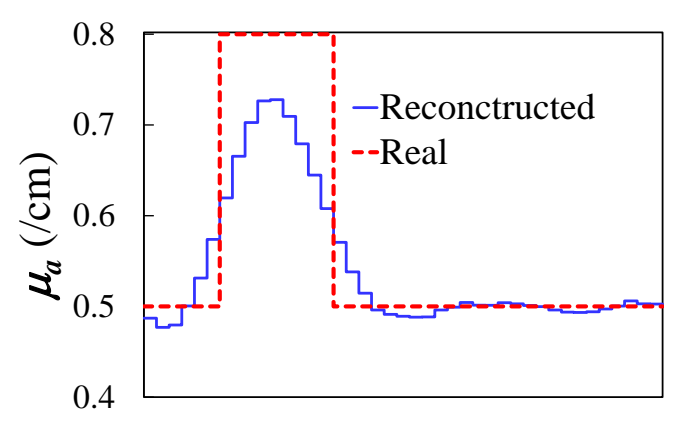

Fig. 4 Real and reconstructed distributions of absorption coefficient along the diagonal line from the bottom-left corner to the top-right corner (case for single inclusion). 


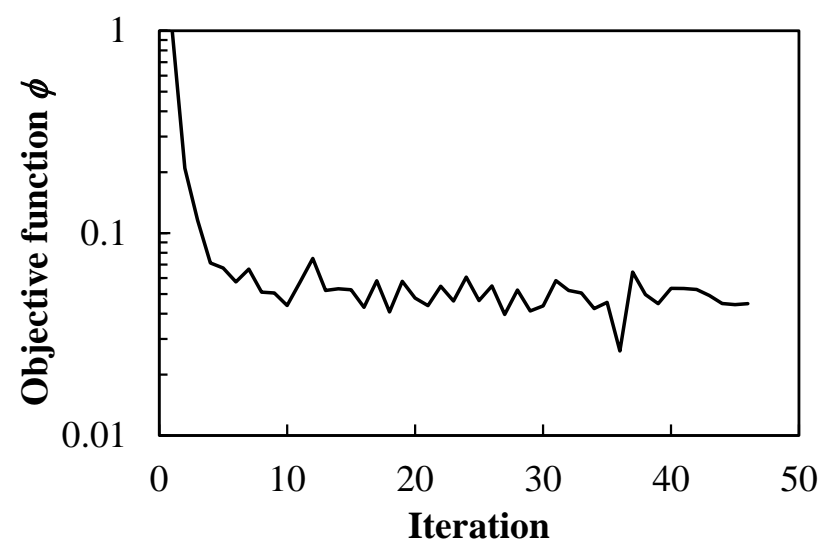

Fig.5 Objective function versus outer iteration for single inclusion and retrieval of absorption coefficient only. 


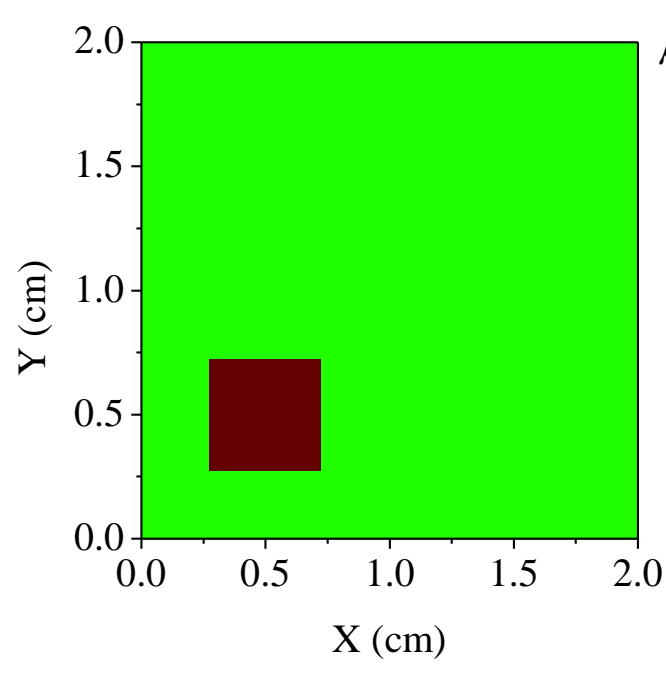

(a)

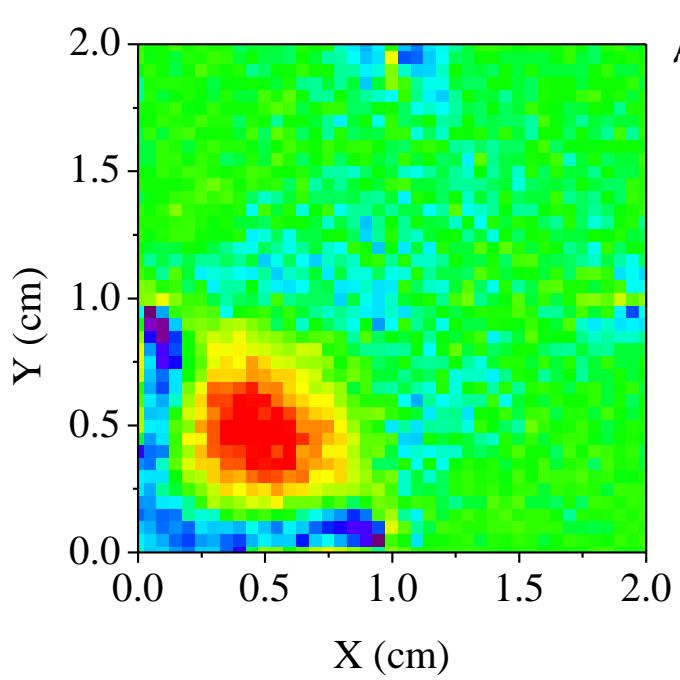

(c)
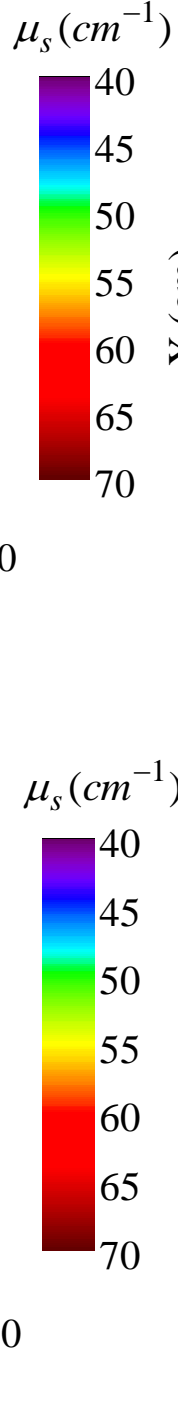

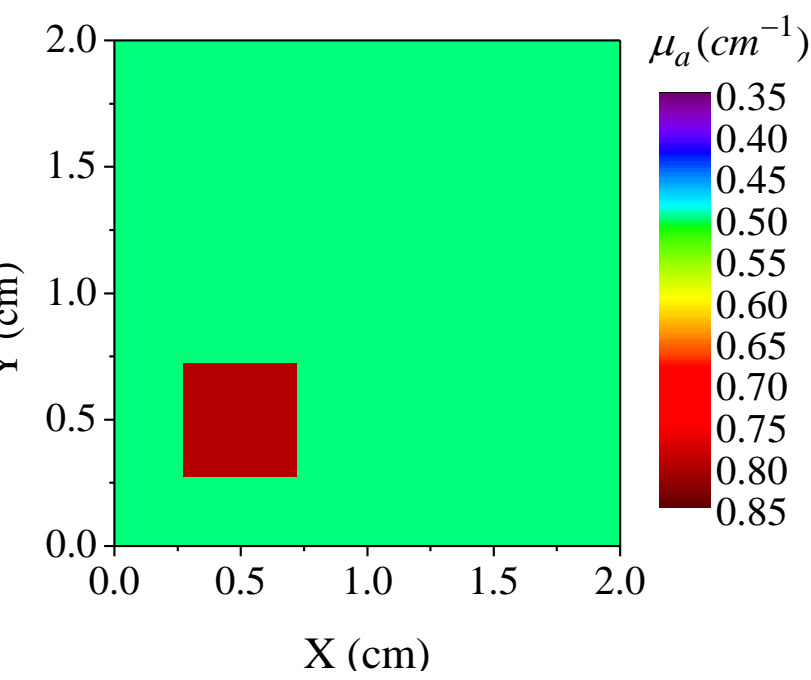

(b)

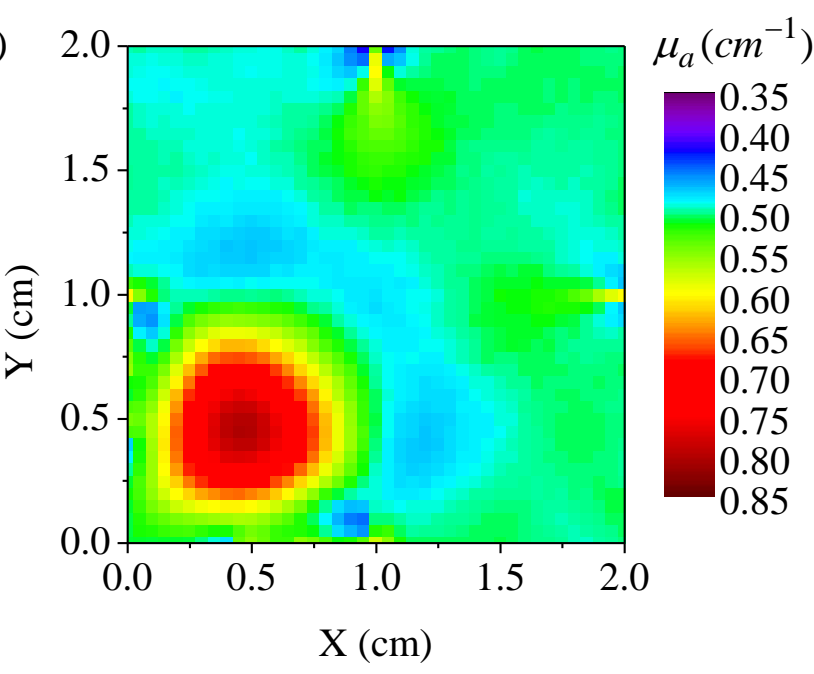

(d)

Fig. 6 Distribution of scattering and absorption coefficients. (a), (b): real distributions. (c), (d): reconstructed distributions. 


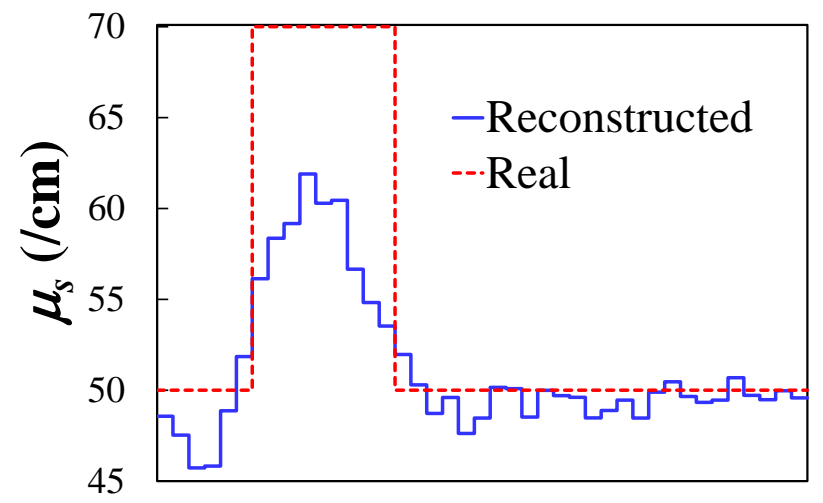

(a)

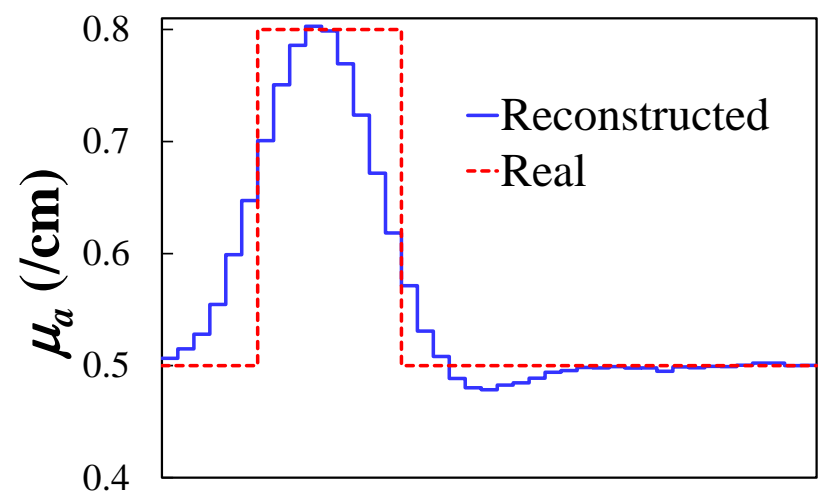

(b)

Fig. 7 Real and reconstructed distributions of scattering coefficient (a) and absorption coefficient (b) along the diagonal line from the bottom-left corner to the top-right corner (case for single inclusion). 


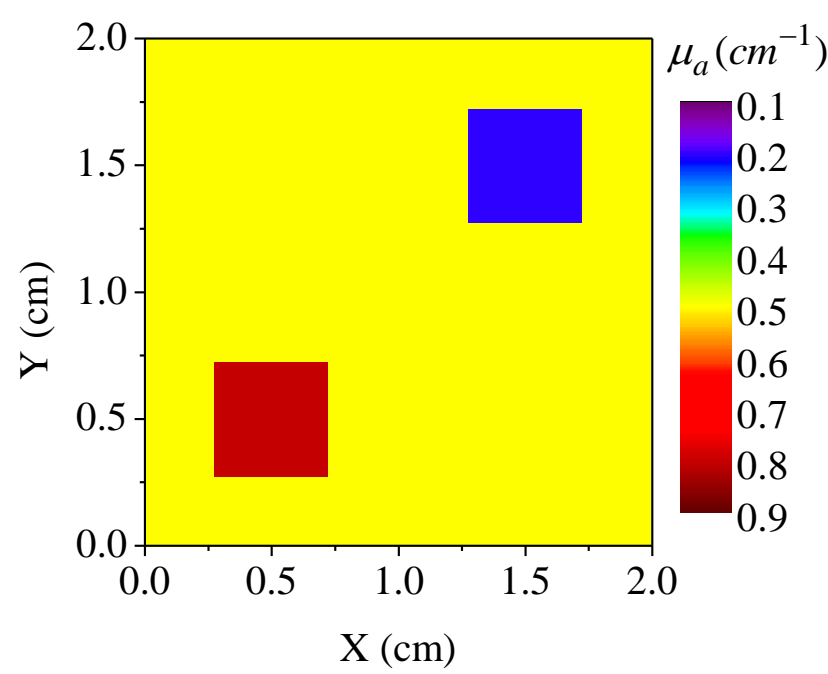

(a)

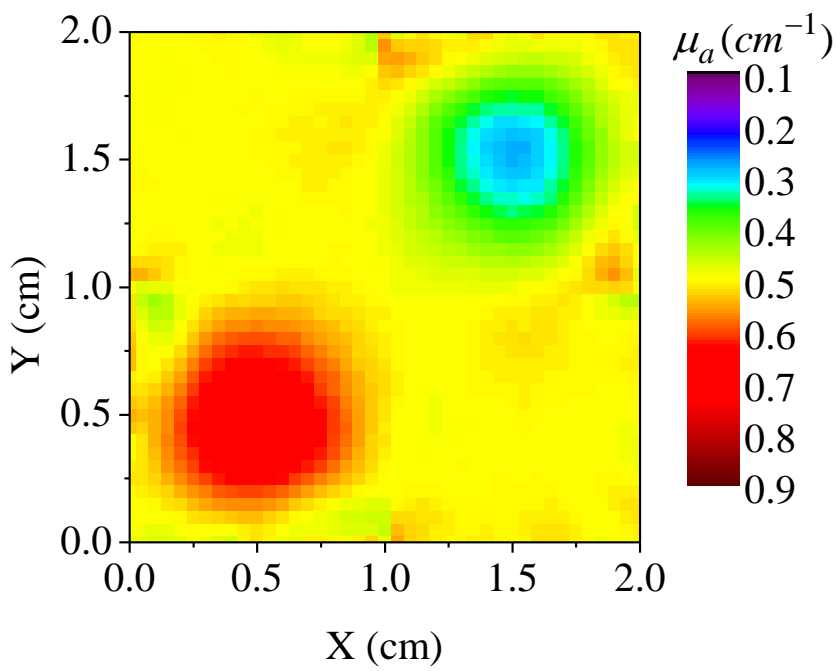

(b)

Fig. 8 Distribution of absorption coefficient. (a) real distribution. (b) reconstructed distribution. 


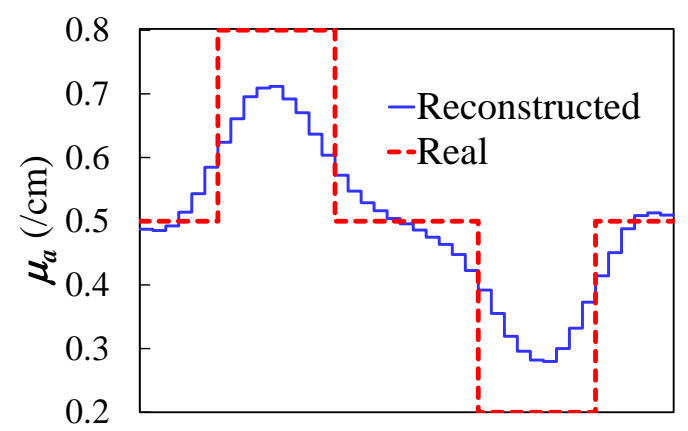

Fig. 9 Real and reconstructed distributions of absorption coefficient along the diagonal line from the bottom-left corner to the top-right corner (case for double inclusions). 


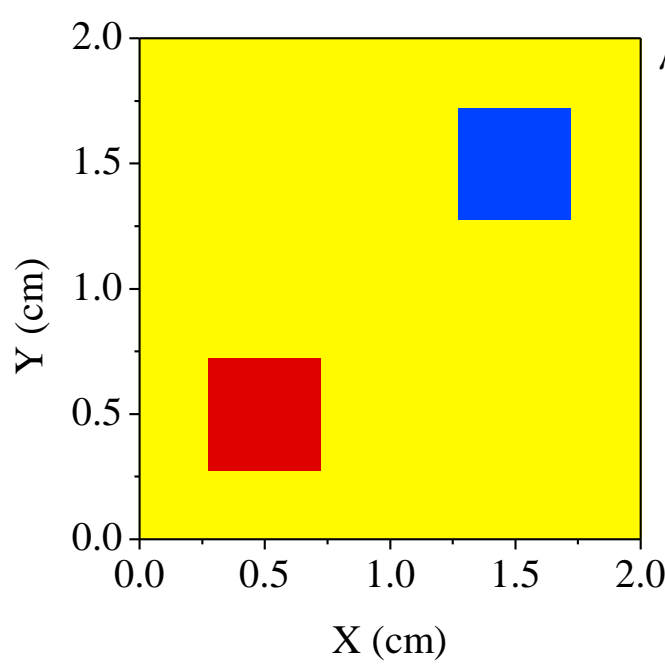

(a)

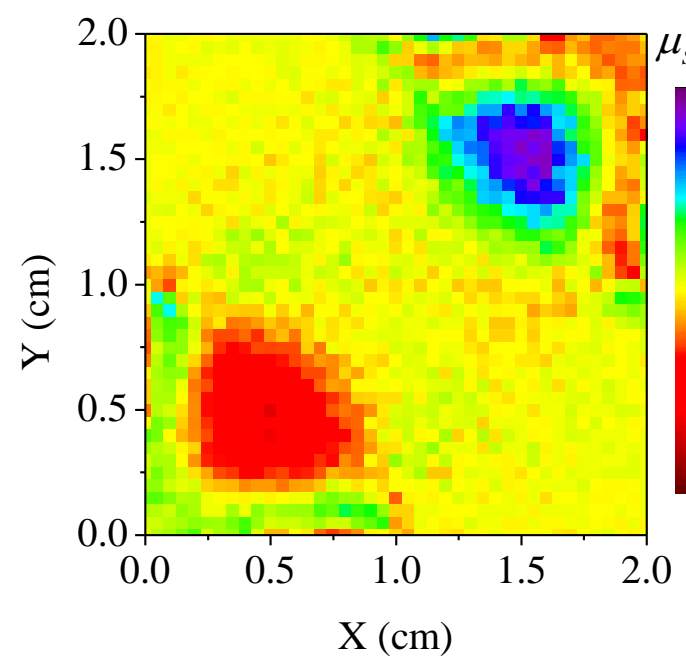

(c)
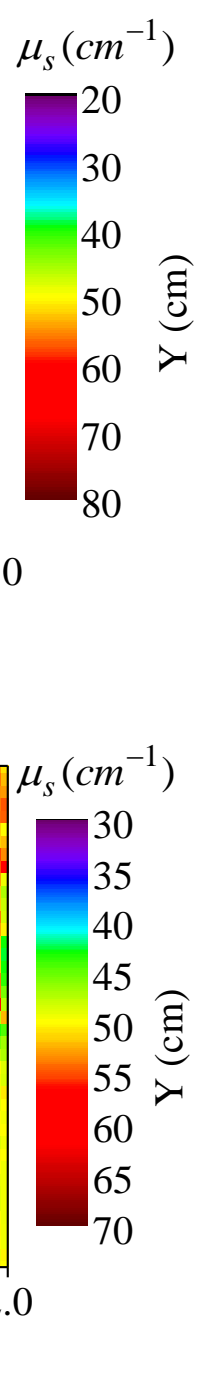

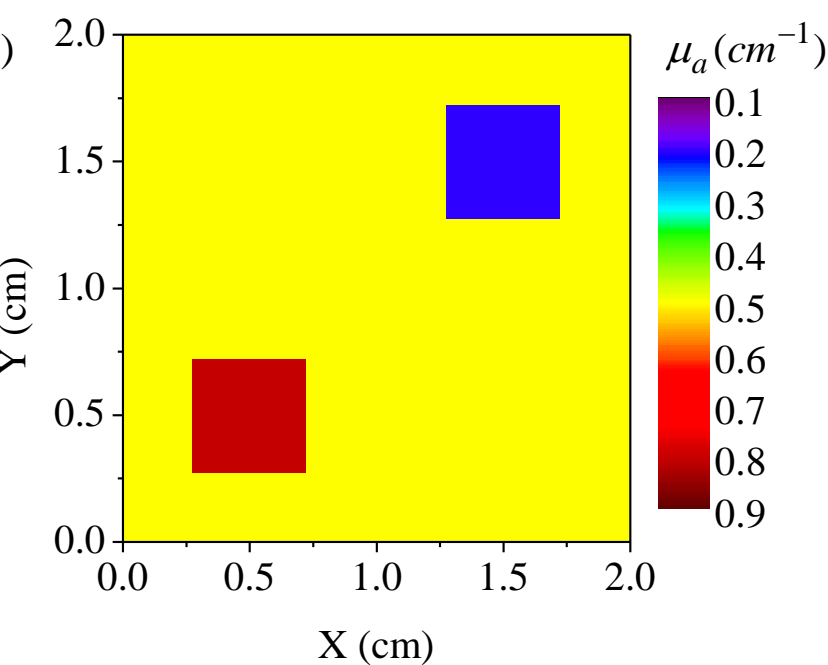

(b)

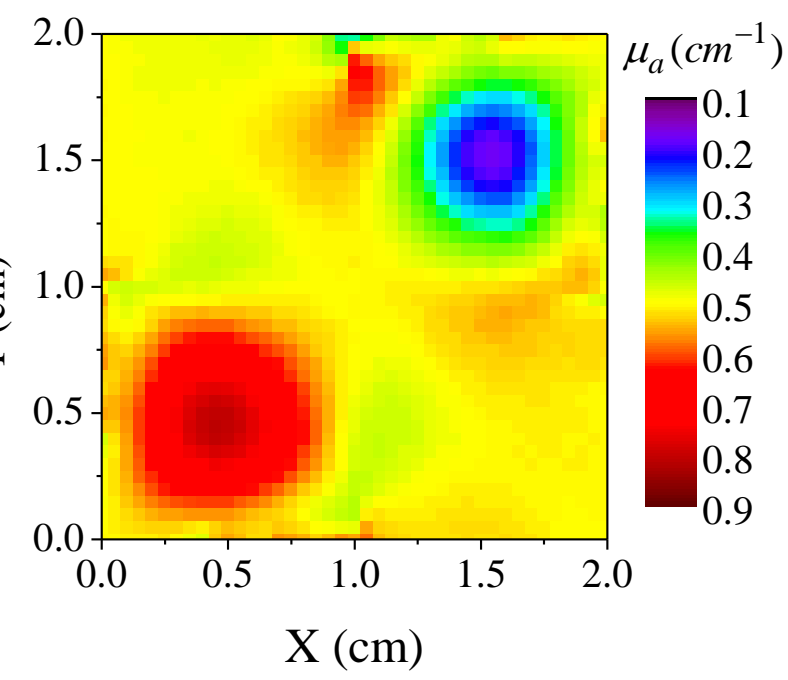

(d)

Fig. 10 Distribution of scattering and absorption coefficients. (a), (b): real distributions. (c), (d) reconstructed distributions (case for double inclusions). 


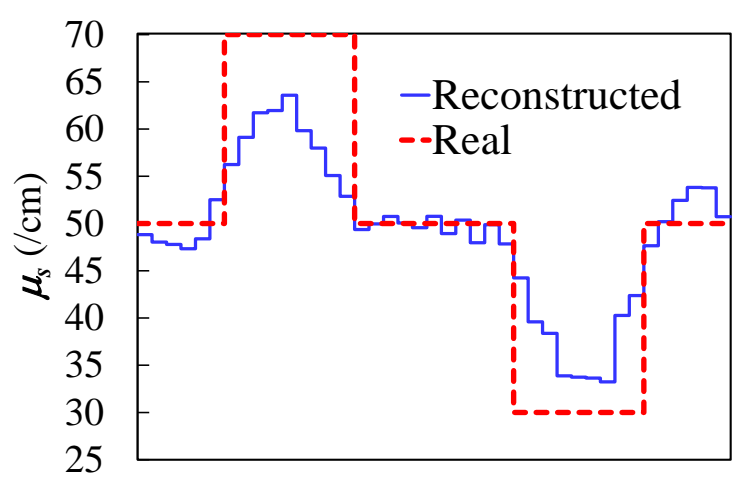

(a)

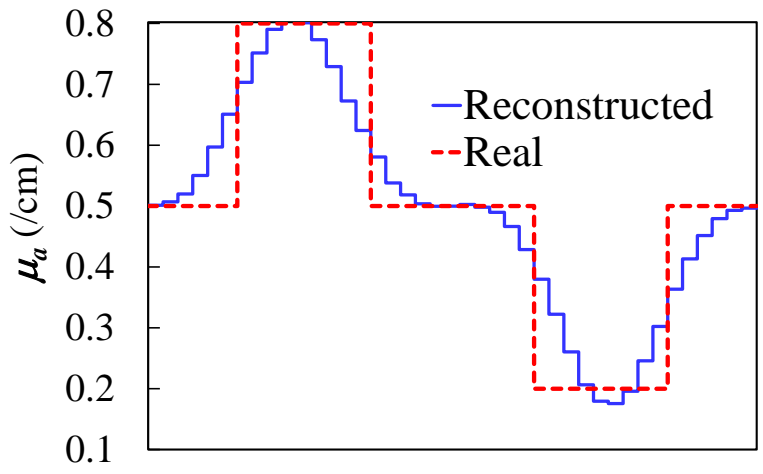

(b)

Fig. 11 Real and reconstructed distributions of scattering coefficient (a) and absorption coefficient (b) along the diagonal line from the bottom-left corner to the top-right corner (case for double inclusions). 


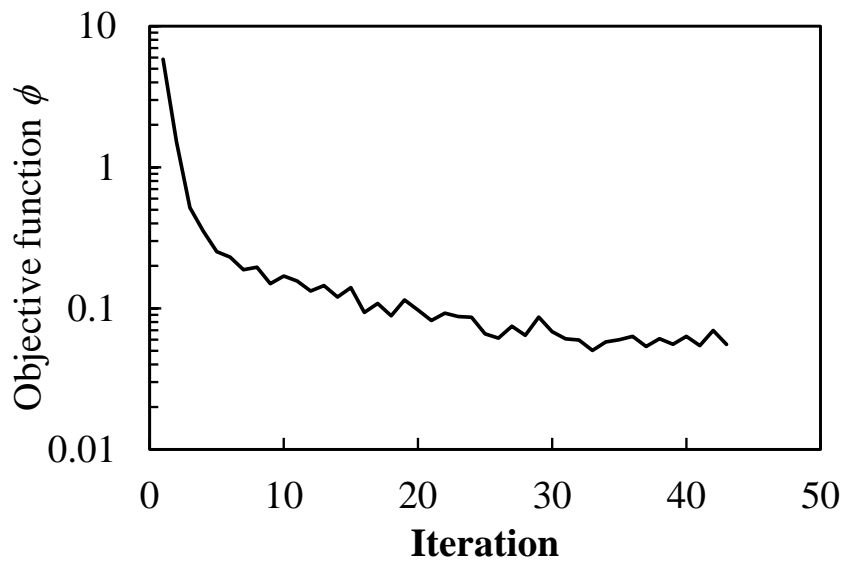

Fig.12 Objective function versus outer iteration for double inclusions and simultaneous retrieval of scattering and absorption coefficients. 


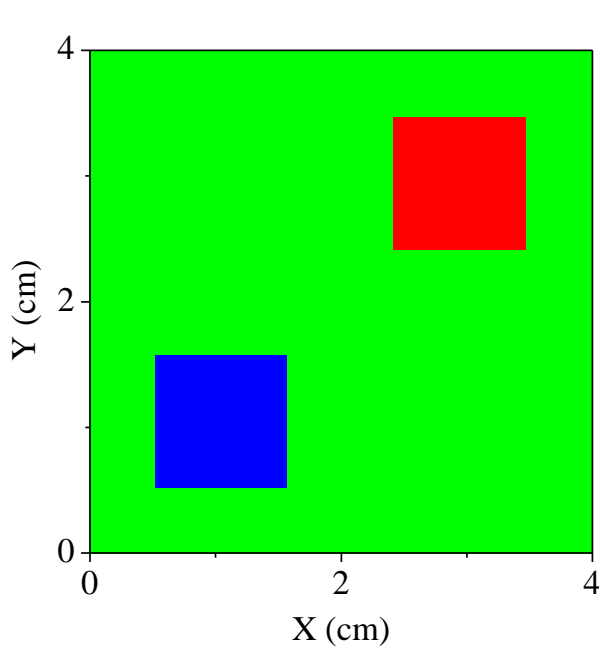

(a)

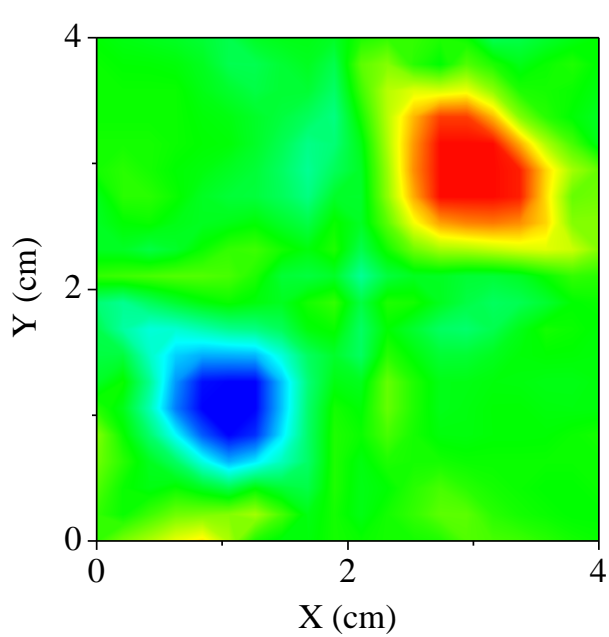

(c)

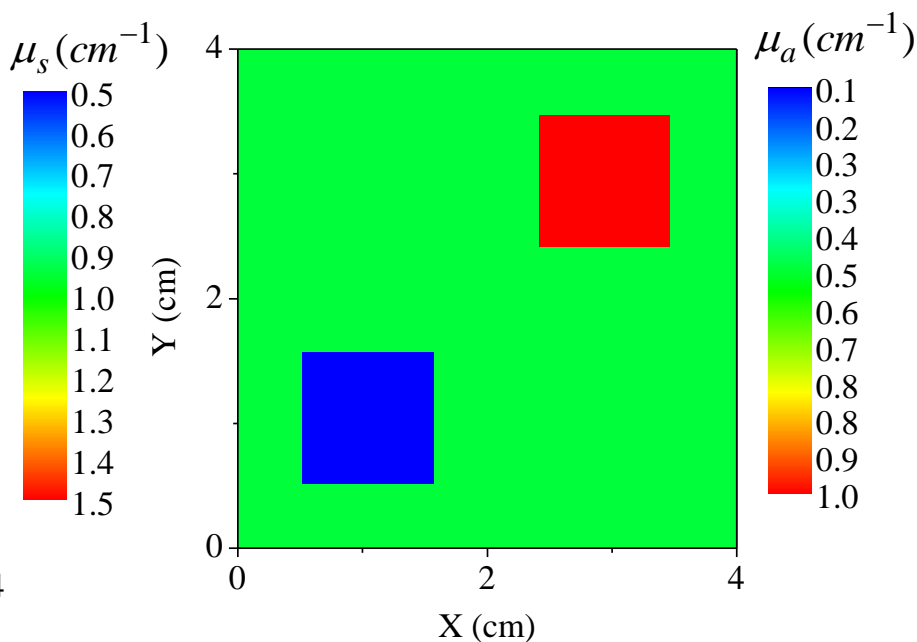

(b)
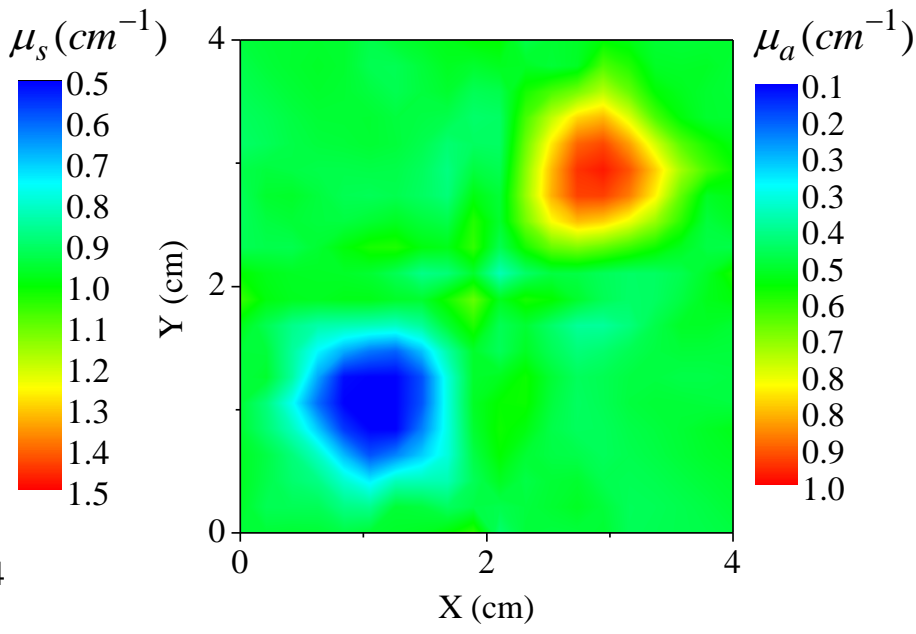

(d)

Fig. 13 Distribution of scattering and absorption coefficients. (a), (b): real distributions. (c), (d) reconstructed distributions (case for Fig. 9 in [13]). 